\title{
1 Photoinduced reactivity in a dispiro-1,2,4-trioxolane: adamantane ring 2 expansion and first direct observation of the long-lived triplet diradical 3 intermediates

13 ABSTRACT

15 The dispiro-1,2,4-trioxolane 1, an ozonide with efficient and broad antiparasitic activity, was 16 synthesized and investigated using matrix isolation FTIR and EPR spectroscopies together 17 with both B3LYP/6-311++G(3df,3dp) and M06-2X/6-311++G(3df,3dp) theoretical 18 methods. Irradiations $\left(\lambda \geq 290 \mathrm{~nm}\right.$ ) of the matrix isolated 1 (Ar or $\mathrm{N}_{2}$ ) afforded exclusively 19 4-oxahomoadamantan-5-one 4 and 1,4-cyclohexanedione 5. These results suggested that the 20 reaction proceeded via a dioxygen-centered diradical intermediate, formed upon homolytic 21 cleavage of the labile peroxide bond, which regioselectively isomerized to form the more 22 stable (secondary carbon-centered)/oxygen-centered diradical. In situ EPR measurements 23 during the photolysis of $\mathbf{1}$ deposited in a MeTHF-matrix led to the detection of signals 24 corresponding to two triplet species, one of which was short-lived while the other proved to 25 be persistent at $10 \mathrm{~K}$. These observations strongly support the proposed mechanism for the 26 photogeneration of $\mathbf{4}$ and $\mathbf{5}$, which involves intramolecular rearrangement of the intermediate 27 diradical species $\mathbf{2}$ to afford the triplet diradical $\mathbf{3}$.

$29 *$ Corresponding authors e-mails:

30 R.F.: rfausto@ci.uc.pt

31 M.L.S.C.: mcristi@ualg.pt 


\section{INTRODUCTION}

Organic dispiro-endoperoxides, in particular dispiro 1,2,4-trioxanes and 1,2,4trioxolanes (ozonides), have been attracting much attention since the discovery of artemisinin more than 40 years ago ${ }^{1-3}$, which provided a completely new antimalarial structural prototype of pharmacophore. The mechanism of bioactivation and action of the artemisinins and related endoperoxide based drugs has been discussed thoroughly ${ }^{4-14}$ and bioactivation is known to require iron(II)-induced reductive cleavage of the peroxide bond to form oxygen-centered radicals, followed by rearrangement to generate carbon-centered radical species that act through modification of parasites biological targets. From a synthetic point of view, endoperoxides are useful for preparation of different types of compounds via ring expansion reactions ${ }^{15}$, and also for the synthesis of $\alpha$-keto $\operatorname{acids}^{16}{ }^{c i s-d i o l s}{ }^{17}, 1,2$-diol monoesters ${ }^{18,19}$, benzofurans and benzopyrans ${ }^{20}$ via selective ring cleavage.

In all the above chemical processes, radical or diradical species have been hypothesized to be the key reactive intermediates, following the initial homolytic cleavage step. For asymmetrically substituted dispiro-1,2,4-trioxolanes, represented by compound 1, and also for their dispiro-1,2,4-trioxane analogues, it has been postulated, based on the final reaction products obtained, that after the cleavage of the peroxide bond generating the dioxygencentered diradical 2 a regioselective $\beta$-scission occurs concomitantly with the rearrangement, leading to the carbon-centered/oxygen-centered diradical species $\mathbf{3}$ (see Scheme 1) ${ }^{21-23}$.

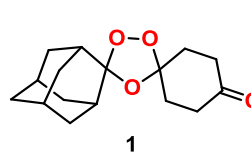

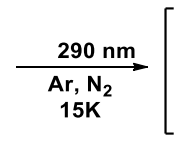

$5 \mathrm{~K}$

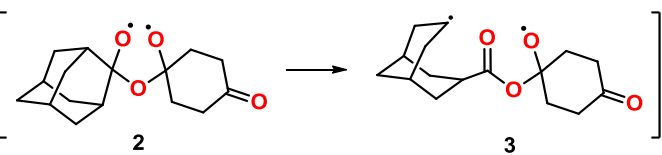

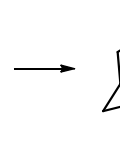

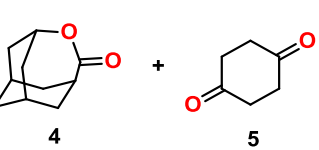

54 Scheme 1. Generic mechanistic scheme for a representative reaction of a dispiro-1,2,4-trioxolane starting with the homolytic cleavage of the $\mathrm{O}-\mathrm{O}$ bond. The example chosen relates with the present study, where the reaction took place in a cryogenic matrix (either solid $\operatorname{Ar}$ or $\mathrm{N}_{2}$, at $15 \mathrm{~K}$ ), the substituents of the trioxolane core being the spiroadamantyl and spiro- $p$-cyclohexanonyl groups and using UV light $(\lambda \geq 290 \mathrm{~nm})$ as the reaction initiator.

When Fe(II) is involved in the reaction, as during the Fe(II)-induced cleavage of the antimalarial peroxides, the metal ion binds to the radical species ${ }^{4-14}$. In spite of the 
62 accumulated evidence of the participation of radicals or diradicals in all these types of

63 reactions, and also of the successful detection of some of these species for reactions involving

64 cyclic peroxides $^{24,25}$, the experimental detection and characterization of the diradical species

65 following the homolytic peroxide bond cleavage of dispiro-1,2,4-trioxolanes has not been 66 reported hitherto.

67 In the present article, we report the first direct observation of the two claimed diradical 68 intermediates for this type of reaction. The strategy used to achieve this goal started with the 69 identification of the final products of the UV-induced unimolecular photolysis of 70 adamantane-2-spiro-3'-8'-oxo-1',2',4'-trioxaspiro[4,5]decane (1) isolated in low 71 temperature matrices (argon and $\mathrm{N}_{2}$ ), and their vibrational characterization. Subsequently, 72 the EPR spectroscopy was used to search for the involved diradicals generated under UV73 photolysis of the compound isolated in a low temperature MeTHF matrix. The choice for the 74 low temperature solid state media to perform this investigation was motivated by three main 75 reasons: (1) under these conditions, because the molecules of the precursor are cage-confined 76 and diffusion does not take place ${ }^{26-30}$, no products can be expected to be formed resulting 77 from species produced from fragmentation of different molecules; this considerably 78 simplifies the accessible chemistry, thus allowing to focus on the main features of the 79 mechanism of the reaction; (2) due to the low work temperature (of a few K) the attainable 80 spectral resolution is much higher compared to what can be obtained, for example, in 81 solution ${ }^{26-30}$; together with absence of significant interactions with the host material this 82 feature allowed to obtain data of better quality for structural elucidation and, at the same 83 time, that could be directly compared with results of quantum chemical computational 84 predictions, which were done for the isolated molecules in vacuo; (3) finally, it could also be 85 expected that the lifetime of the intermediates were longer under the used experimental 86 conditions (low temperature; inhibited molecular diffusion; relative inertness of the 87 medium), so that their experimental detection would be facilitated.

88 As described in detail below, the obtained results demonstrate that the UV-induced 89 photolysis of the investigated dispiro-1,2,4-trioxolane proceeds regioselectively, leading to 90 its adamantane substituent ring expansion, via the two key diradical triplet species suggested 91 previously, which could be successfully detected here for the first time by EPR spectroscopy. 92 As it could be expected, the measured lifetimes of the two diradicals differ considerably, with 
93 the carbon-centered/oxygen-centered diradical being considerably more stable than its 94 oxygen centered diradical precursor.

95 It is worth noting that trioxolane $\mathbf{1}$ has very relevant and broad antiparasitic properties. It

96 has shown low nanomolar activity against Plasmodium falciparum $^{31}$ and recent studies

97 demonstrated that its activity is retained against $P$.falciparum strains resistant to chloroquine 98 and to the artemisinin-derived drugs used in combination therapies (ACT) ${ }^{32}$. Compound 1 99 also exhibits low micromolar activity against intracellular amastigote forms of Leishmania 100 infantum $^{33}$ and against Perkinsus olseni $^{34}$. In addition to its broad antiparasitic activity, the 101 studied compound can be prepared from the commercially available 2-adamantanone and 102 1,4-cyclohexanedione building blocks in only 2 steps.

103

\section{EXPERIMENTAL AND COMPUTATIONAL DETAILS}

\section{General}

Commercial reagents were used as purchased. ${ }^{1} \mathrm{H}$ and ${ }^{13} \mathrm{C}-\mathrm{NMR}$ spectra were recorded on a $400 \mathrm{MHz}$ NMR spectrometer Bruker Avance III 400. ${ }^{1} \mathrm{H}-\mathrm{NMR}$-chemical shifts are referred to the residual signal of $\mathrm{CDCl}_{3}(\delta \mathrm{H} 7.26)$ and ${ }^{13} \mathrm{C}-\mathrm{NMR}$-chemical shifts to the $\mathrm{CDCl}_{3}$ signal ( $\delta \mathrm{C} 77.0)$, or using TMS as internal standard. Thin-layer chromatography was carried out on silica gel 60 F254 plates (AL TLC 20x20). Column chromatography was performed on Silica Gel $60(0.04-0.063 \mathrm{~mm})$. Melting points $\left({ }^{\circ} \mathrm{C}\right)$ were obtained on a SMP3 Melting Point Apparatus and are uncorrected.

\section{Syntheses and general characterization of the compounds}

Adamantane-2-spiro-3'-8'-oxo-1',2',4'-trioxaspiro[4,5]decane 1: ozone, produced with an ozone generator Sander Labor-Ozonizator $301.7\left(0.5 \mathrm{~L} / \mathrm{min} \mathrm{O}_{2}, 140 \mathrm{~V}\right)$, was passed through a solution of dichloromethane at $-78{ }^{\circ} \mathrm{C}$ and flushed into a solution of $O$-methyl 2adamantanone oxime $(1.00 \mathrm{~g}, 5.58 \mathrm{mmol})$ and 1,4-cyclohexanedione $(0.65 \mathrm{~g}, 5.58 \mathrm{mmol})$ in pentane $(60 \mathrm{~mL})$ and dichloromethane $(40 \mathrm{~mL})$ at $0{ }^{\circ} \mathrm{C}$. After consumption of the starting material, the solution was flushed with nitrogen for $5 \mathrm{~min}$ and concentrated under reduced pressure at room temperature to give a crude material. Purification by flash chromatography using a mixture of EtOAc/n-hexane, gave the pure compound as a colorless solid $(0.66 \mathrm{~g}$, $42 \%$ yield): m.p. $127-128{ }^{\circ} \mathrm{C} ;{ }^{1} \mathrm{H}-\mathrm{NMR}\left(400 \mathrm{MHz}, \mathrm{CDCl}_{3}\right): \delta 1.69-2.02(\mathrm{~m}, 14 \mathrm{H}), 2.14(\mathrm{t}, J$ 
$124=6.9 \mathrm{~Hz}, 4 \mathrm{H}), 2.51(\mathrm{t}, J=7.0 \mathrm{~Hz}, 4 \mathrm{H}) \mathrm{ppm} ;{ }^{13} \mathrm{C}-\mathrm{NMR}\left(100 \mathrm{MHz}, \mathrm{CDCl}_{3}\right): 25.9,26.31,31.09$, $12532.59,34.25,35.70,36.18,37.35,106.46,111.95,208.90$ ppm; MS (EI, $m / z): 278.9[\mathrm{M}]^{+}$.

126 The $O$-methyl 2-adamantanone oxime precursor of compound 1 was obtained as follows: 127 to a solution of 2-adamantanone (4.00 g, $26.63 \mathrm{mmol})$ in methanol $(20 \mathrm{~mL})$, under stirring, 128 was added pyridine $(3.40 \mathrm{~mL}, 42.03 \mathrm{mmol})$ and methoxylamine hydrochloride $(2.846 \mathrm{~g}$, $12934.08 \mathrm{mmol}$ ). The reaction mixture was stirred at room temperature for $72 \mathrm{~h}$. The final 130 mixture was concentrated and then diluted with dichloromethane $(20 \mathrm{~mL})$ and water $(30 \mathrm{~mL})$.

131 The organic layer was separated, and the aqueous layer was washed with dichloromethane 132 (2x20 mL). The combined organic layers were washed with aqueous $\mathrm{HCl}(1 \mathrm{M} ; 20 \mathrm{~mL} \times 2)$, 133 then with brine $(20 \mathrm{~mL})$. The final organic extract was dried with $\mathrm{MgSO}_{4}$, filtered, then 134 concentrated under reduced pressure to give $O$-methyl-2-adamantanone oxime (4.20 g, 88\% 135 yield) as a colorless solid (m.p. 69-70 ${ }^{\circ} \mathrm{C}$ ). ${ }^{1} \mathrm{H}-\mathrm{NMR}\left(400 \mathrm{MHz}, \mathrm{CDCl}_{3}\right.$ ): $\delta 3.81$ (s, 3H), 3.46 136 (s,1H), $2.54(\mathrm{~s}, 1 \mathrm{H}), 2.00-1.78(\mathrm{~m}, 12 \mathrm{H}) \mathrm{ppm} .{ }^{13} \mathrm{C} \mathrm{NMR}\left(101 \mathrm{MHz}, \mathrm{CDCl}_{3}\right) \delta: 166.74$, $13760.96,39.03,37.64,36.52,36.24,29.53,27.85$ ppm. MS (MALDI-TOF, $m / z): 180.02[\mathrm{M}]^{+}$.

138 4-Oxahomoadamantan-5-one 4, was synthesized using the procedure described by 139 Renoud-Grappin et al..$^{35}$ To a suspension of 2-adamantanone $(0.5 \mathrm{~g}, 3.33 \mathrm{mmol})$ and $\mathrm{NaHCO}_{3}$ 140 (0.31 g, $3.66 \mathrm{mmol})$ in anhydrous dichloromethane $(10 \mathrm{~mL})$ was added a solution of $m$ 141 chloroperoxybenzoic acid ( $m$-CPBA) (0.86 g, $4.99 \mathrm{mmol})$ in anhydrous dichloromethane (5 $142 \mathrm{~mL}$ ). The reaction mixture was stirred at room temperature, in the dark, until consumption of 143 the starting compound. Then the organic mixture was washed with water $(3 \times 15 \mathrm{~mL})$, brine $144(2 \times 15 \mathrm{~mL})$ and dried over with $\mathrm{MgSO}_{4}$. The organic layer was then evaporated to dryness 145 under reduced pressure. Purification of the residue by flash chromatography using a mixture 146 of EtOAc/ $n$-hexane, gave the required product as a white solid ( $0.35 \mathrm{~g}, 63 \%$ yield): m.p: 288 147 $290{ }^{\circ} \mathrm{C} .{ }^{1} \mathrm{H}$ NMR $\left(400 \mathrm{MHz}, \mathrm{CDCl}_{3}\right): \delta=4.49(\mathrm{tt}, J=4.4,2.4 \mathrm{~Hz}, 1 \mathrm{H}), 3.10-3.05(\mathrm{~m}, 1 \mathrm{H})$, $14930.96,25.85$. HRMS (CI, $m / z)$ calcd for $\mathrm{C}_{10} \mathrm{H}_{15} \mathrm{O}_{2}(\mathrm{M}+\mathrm{H})^{+}:$: 167.1067; found 167.1072. 1,4-Cyclohexanedione 5 was purchased from Sigma Aldrich UK and used without 151 further purification.

152 All compounds prepared were kept in the freezer and shielded from the light during 153 storage, remaining stable under these conditions. 
To prepare the low temperature matrices a sample of the solid compound to be studied was placed in an especially designed thermoelectrically heatable mini-oven attached to the vacuum chamber of a helium cryostat (APD Cryogenics closed-cycle helium refrigerator system with a DE-202A expander). Before the measurements, the samples were subjected to additional purification by continued high-vacuum ( $\sim 10^{-7}$ mbar $)$ pumping, during approximately 1 hour, at room temperature. The samples were then sublimed and the vapors

162 of the compound to be studied were deposited, together with a large excess of argon or $\mathrm{N}_{2}$, onto a cesium iodide (CsI) substrate mounted at the cryostat cold tip $(15 \pm 0.1 \mathrm{~K}$, as measured

164 by a silicon diode sensor connected to a Scientific Instruments digital temperature controller).

165 This temperature was kept during the overall experiments.

166 The IR spectra of the matrix-isolated compounds were recorded in the $400-4000 \mathrm{~cm}^{-1}$ range and with $0.5 \mathrm{~cm}^{-1}$ spectral resolution, using a Nicolet 6700 Fourier transform infrared (FTIR) spectrometer, equipped with a deuterated triglycine sulfate (DTGS) detector and a $\mathrm{Ge} / \mathrm{KBr}$ beam splitter. The optical bench was continuously purged with a flux of dry and $\mathrm{CO}_{2}$ filtered $\mathrm{N}_{2}$, to avoid interference from atmospheric $\mathrm{H}_{2} \mathrm{O}$ and $\mathrm{CO}_{2}$. $\left(\sim 3.0 \times 10^{-2} \mathrm{~Pa}\right)$ in a quartz EPR tube, which was sealed after three freeze-pump-thaw cycles under the vacuum conditions. The MeTHF solution of compound 1 was irradiated in the EPR cavity at $10 \mathrm{~K}$. The X-band EPR signals were obtained at a resonance frequency of $9.40 \mathrm{GHz}$ using a Bruker E500 spectrometer at 10-50 K.

Matrix isolation EPR spectroscopy measurements

A $100 \mathrm{mM}$ solution of compound 1 in $\operatorname{MeTHF}(100 \mu \mathrm{L})$ was degassed under high vacuum

\section{In situ UV irradiation experiments}

In the steady state infrared spectroscopy experiments carried out in $\mathrm{Ar}$ and $\mathrm{N}_{2}$ matrices, in situ $\mathrm{UV}$ irradiation of the samples was undertaken using different approaches. In the case of the experiments carried out in the Ar matrices, broadband UV radiation was used, as provided by the $\mathrm{KBr}$ external window of the cryostat and water filtered $(\geq 290 \mathrm{~nm}$ ) light generated by a $500 \mathrm{~W} \mathrm{Hg}(\mathrm{Xe})$ lamp (Newport, Oriel Instruments), with output power set to $200 \mathrm{~W}$. For the experiments carried out in the $\mathrm{N}_{2}$ matrices, tunable narrowband light was used ( $\sim 0.1 \mathrm{~cm}^{-1}$ bandwidth; $\lambda=290 \mathrm{~nm}$; pulse energy $\left.\sim 8 \mathrm{~mJ}\right)$, provided by a Spectra Physics 
186 Quanta-Ray MOPO-SL optical parametric oscillator (OPO) pumped with a pulsed Nd:YAG 187 laser (repetition rate $=10 \mathrm{~Hz}$, duration $=10 \mathrm{~ns}$ ).

188 For the EPR measurements, the photolysis was conducted at $266 \mathrm{~nm}(5 \mathrm{~mJ})$, using a Nd189 YAG laser (Spectra Physics Indi-40).

\section{Computational Details}

192 Quantum chemistry computations were performed at both the B3LYP/6193 311++G(3df,3dp) and M06-2X/6-311++G(3df,3dp) levels of theory ${ }^{36-43}$, using Gaussian 09 194 (Revision D.01) ${ }^{44}$. Geometry optimizations were performed using the "tight" optimization 195 criteria.

196 The harmonic vibrational wavenumbers and IR intensities were calculated at the same 197 levels of theory and scaled in order to correct them for the neglect of anharmonicity, basis 198 sets restraints and the effect of incomplete treatment of the electron correlation. The scaling 199 factors were acquired by fitting the computed harmonic to the experimental IR wavenumbers 200 of compound 1 within the 1900-600 $\mathrm{cm}^{-1}$ region and those of compounds 4 and 5 within the 201 1900-500 $\mathrm{cm}^{-1}$ (see Figure S1 in the Supporting Information). The slopes obtained by least202 squares linear fit, intercepting zero $(y=b x)$, were then used to scale the computed harmonic 203 wavenumbers of the photoproducts and reactant in the 1900-400 $\mathrm{cm}^{-1}$ range. The simulation 204 of the IR spectra was achieved using the calculated (scaled) wavenumbers and IR intensities 205 (in $\mathrm{km} \mathrm{mol}^{-1}$ ), which were convoluted with Lorentzian functions with a full-width at half206 maximum (fwhm) of $2 \mathrm{~cm}^{-1}$. conformers of the compound with similar energies (see Table 1), which are represented in

214 Figure 1. The two conformers differ in the orientation of the spiro- $p$-cyclohenanonyl moiety, 215 which in the most stable form (A) is turned to the peroxide group and in the less stable one 216 (B) is oriented towards the ring ether fragment. Their population ratio (A:B) in the room 
217 temperature $(298.15 \mathrm{~K})$ gas phase equilibrium is expected to stay between 1.1 and 1.5 (which 218 are the values resulting from the relative energies obtained in the B3LYP and M06-2X 219 calculations, respectively), i.e., both forms are predicted to be significantly populated in these 220 experimental conditions, and can then be expected to be present in the cryogenic matrices 221 investigated in this work (see below). The Cartesian coordinates of the two conformers, as 222 predicted by the two methods used in this study, are provided as Supporting Information 223 (Tables S1 and S2).

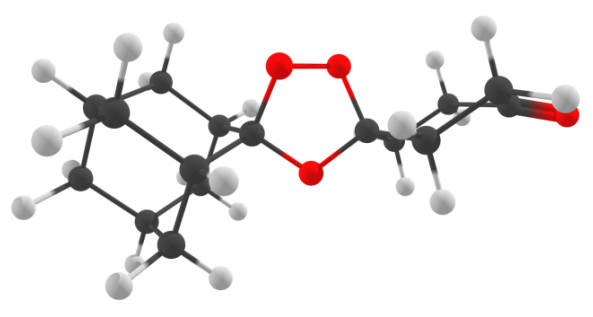

$1 \mathrm{~A}$

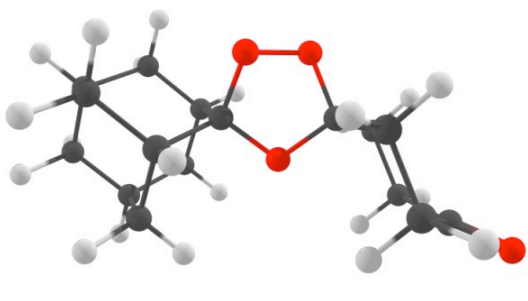

1B
224

225

226

227

228

229

230

Figure 1. B3LYP/6-311++G(3df,3pd) optimized structures of the two conformers of $\mathbf{1}$ in the electronic ground state $\left(\mathrm{S}_{0}\right)$.

Table 1. B3LYP/6-311++G(3df,3pd) and M06-2X/6-311++G(3df,3pd) calculated energies (kJ mol ${ }^{1}$ ) for the conformers of $\mathbf{1}$ (see Figure 1) zero-point energies (ZPE) and estimated room temperature (298.15 K) percent populations in the gas phase equilibrium.

\begin{tabular}{|c|c|c|c|c|}
\hline & \multicolumn{2}{|l|}{ B3LYP } & \multicolumn{2}{|l|}{ M06-2X } \\
\hline & $\mathbf{1 A}$ & 1B & $\mathbf{1 A}$ & 1B \\
\hline Energy & -2426219.58 & -2426218.50 & -2425270.01 & -2425269.82 \\
\hline ZPE & 954.83 & 954.74 & 966.62 & 966.59 \\
\hline Energy (with ZPE) & -2425264.75 & -2425263.76 & -2424303.39 & -2424303.22 \\
\hline$\Delta$ Energy & 0.00 & 0.99 & 0.00 & 0.17 \\
\hline Population (298.15 K) & $59.8 \%$ & $40.2 \%$ & $51.7 \%$ & $48.3 \%$ \\
\hline
\end{tabular}



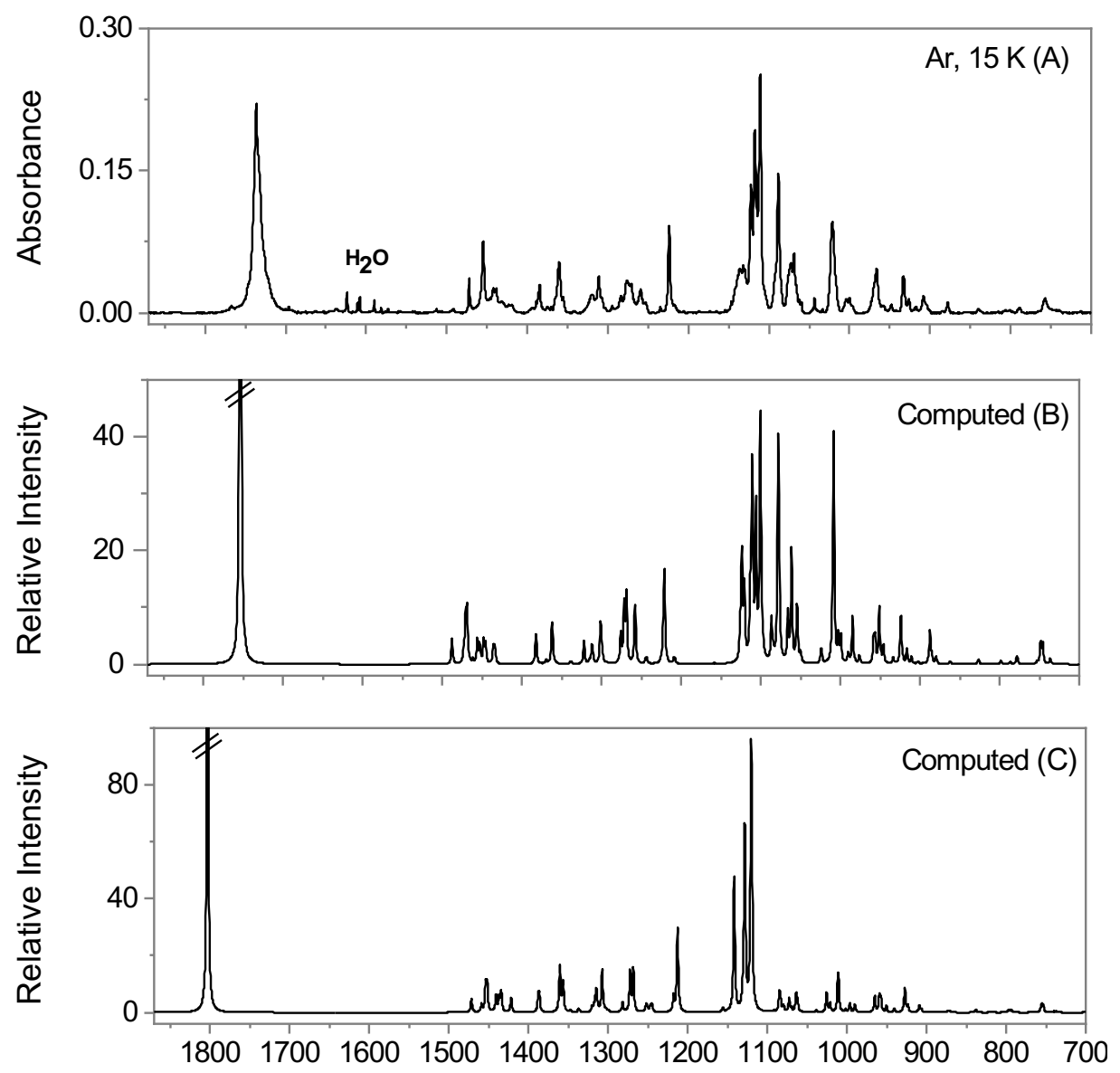

Wavenumber $/ \mathrm{cm}^{-1}$

233 Figure 2. Infrared spectra of 1: (A) isolated in an Ar matrix (15 K); (B), (C) calculated using the 234 B3LYP and M06-2X functionals (with the 6-311+G(3df,3pd) basis set). The calculated spectra are 235 shown as the 1:1 sum of the spectra of both conformers of the compound, and the frequencies were 236 scaled by 0.983 and 0.971 factors in (B) and (C), respectively. The spectrum observed in the $\mathrm{N}_{2}$ matrix 237 is very similar to that obtained in argon and is provided in the Supporting Information (Figure S2).

The dispiro-1,2,4-trioxolane 1 was sublimed under high-vacuum at room temperature and co-deposited with large excess of argon or $\mathrm{N}_{2}$ onto the cold $(15 \mathrm{~K}) \mathrm{CsI}$ substrate of the used cryostat. The infrared spectra of the prepared matrices were subsequently recorded. The 242 spectrum obtained for the compound in the argon matrix is shown in Figure 2, while that 243 obtained in the $\mathrm{N}_{2}$ matrix is provided in the Supporting Information (Figure S2). The two 244 spectra were found to be very similar, testifying the fact that the molecules of the compound 245 were well-isolated in both matrices and also that the conformational population of 1 existing in the gas phase prior to deposition was efficiently trapped in the matrices. Also, the peroxide 
2471 was found not to undergo thermal decomposition upon sublimation under the used 248 conditions for matrix deposition. The spectroscopic data (both experimental and calculated 249 frequencies and intensities) are summarized in Tables S3-S5 (Supporting Information). It can 250 be seen that, in spite of the similarity of the spectra of the two conformers, several bands 251 observed in the experimental spectra could be assigned to the individual forms, thus 252 confirming the presence of both conformers in the matrices.

253 The comparison of the spectrum obtained in the argon matrix with those theoretically 254 predicted (see Figure 2), shows that the B3LYP functional reproduces better the experimental 255 data throughout the spectrum than the M06-2X functional. Thus, spectra calculated using the 256 B3LYP functional will be used in this study for the analysis of the experimentally obtained 257 spectroscopic data of the remaining compounds under study.

258 The UV-induced reactivity of $\mathbf{1}$ was investigated in the two types of matrices used (Ar, $259 \mathrm{~N}_{2}$ ). Argon matrix was used as standard work medium, while $\mathrm{N}_{2}$ matrix, known to stabilize 260 high-energy species by establishing specific interactions with the guest species ${ }^{45,46}$, was 261 chosen to check the possibility of detection in this medium of any putative reaction 262 intermediate. Both broadband $(\lambda \geq 290 \mathrm{~nm})$ and narrowband $(\lambda=290 \mathrm{~nm})$ excitations were 263 applied. The irradiations were performed at wavelengths within the observed band in the UV264 vis spectra of $\mathbf{1}$ in ethanol solution (Figure S3). The results were found to be qualitatively 265 identical in both matrices and for the two excitation procedures followed. Upon excitation, 266 the bands initially present in the spectra reduced in intensity, while new bands started to 267 emerge, indicating conversion of the trioxolane 1 into other chemical species. Particularly 268 noticeable new spectral features were observed in the $v(\mathrm{C}=\mathrm{O})$, (between 1720-1750 $\left.269 \mathrm{~cm}^{-1}\right)$ and $v(\mathrm{C}-\mathrm{O}),\left(1080-1120 \mathrm{~cm}^{-1}\right)$ regions. The intensities of the bands of $\mathbf{1}$ and those of 270 the new bands changed continuously with the irradiation time, until almost complete 271 conversion of the reactant 1 (after $\sim 30 \mathrm{~min}$. of broadband irradiation of the compound in an 272 argon matrix). Detailed analysis of the spectra of the photolysed matrices allowed 273 identification of the observed photolysis products as being 4-oxahomoadamantan-5-one 4 274 and 1,4-cyclohexanedione 5, the expected final products for the photolysis of the trioxolane $275 \mathbf{1}$, initiated by homolytic cleavage of the labile peroxide bond. This can be clearly seen in 276 Figure 3, where the experimental difference IR spectrum (spectrum obtained after $30 \mathrm{~min}$. of 277 irradiation at $\lambda \geq 290 \mathrm{~nm}$ minus spectrum of the as-deposited argon matrix) is compared with 
278 the B3LYP/6-311++G(3df,3pd) calculated difference IR spectrum generated by subtracting 279 the calculated spectrum of $\mathbf{1}$ from the sum of the spectra of $\mathbf{4}$ and $\mathbf{5}$ \{in a ratio $280(0.5[4]+0.5[5]): 1[1]\}$. The identification of the photoproducts was further confirmed by 281 recording the IR spectra of genuine samples of $\mathbf{4}$ and $\mathbf{5}$ (see experimental section) deposited 282 in argon matrices and (i) comparing these spectra with the spectra of the photoproducts 283 generated upon UV irradiation of matrix isolated 1 (see Figure 4), or (ii) comparing the 284 difference experimental IR spectrum shown in Figure 3 (irradiated minus as-deposited matrix 285 spectra) with the one obtained by subtracting the experimental spectrum of $\mathbf{1}$ from the sum 286 of the experimentally obtained IR spectra of the genuine samples of $\mathbf{4}$ and $\mathbf{5}$ isolated in argon 287 matrices (see Figure 5).

288 The structural computed data obtained with the B3LYP and M06-2X functionals for 4 289 and 5 (graphical representation of the optimized minimum energy structures, and summary 290 of calculated energy data), as well as the spectroscopic data obtained both theoretically and 291 experimentally for these compounds (graphical comparison of the calculated and matrix 292 isolation experimental infrared spectra of the compounds, and tables with calculated and 293 experimental frequencies and intensities, those latter including the bands of $\mathbf{4}$ and $\mathbf{5}$ both in 294 the photolysed matrices of $\mathbf{1}$ and as isolated species) are given in the Supporting Information 295 (Tables S6-S14 and Figures S4 and S5). The B3LYP computed vibrational frequencies of 4 and 2965 were scaled by 0.983 and 0.982 , respectively.

297 According to the obtained results, the diradical rearrangement is regioselective, with no 298 evidence of formation of the alternative products, 2-adamantanone and oxocane-2,7-dione. 299 This can be clearly seen when one compares the infrared spectra of the photoproduced 300 species, generated after UV irradiation of the matrix-isolated 1, with those of 2301 adamantanone and oxocane-2,7-dione (see Figure 3; additional calculated structural and 302 spectroscopic data on these two compounds are presented in the Supporting Information, 303 Figure S6 and Tables S15 and S16). The observed regioselectivity can be explained by the 304 expected greater stability of the (secondary carbon-centered)/oxygen-centered diradical 305 species $\mathbf{3}$, in comparison with the putative alternative (primary carbon-centered)/oxygen306 centered diradical that would lead to the non-observed final products. In addition, spin 307 density calculations (performed at the B3LYP/6-311++G(3df,3dp) level) on the triplet state 308 dioxygen-centered diradical result in a considerable larger electron spin density in the tertiary 
$309 \beta$-carbon of the spiroadamantyl substituent as compared to that of the secondary $\beta$-carbon of 310 the spiro-p-cyclohexanonyl substituent, which can also be considered an indication of a most 311 favorable rearrangement of the initially generated dioxygen-centered diradical into the 312 (secondary carbon-centered)/oxygen-centered diradical species $\mathbf{3}$, leading to the observed 313 final products.

314

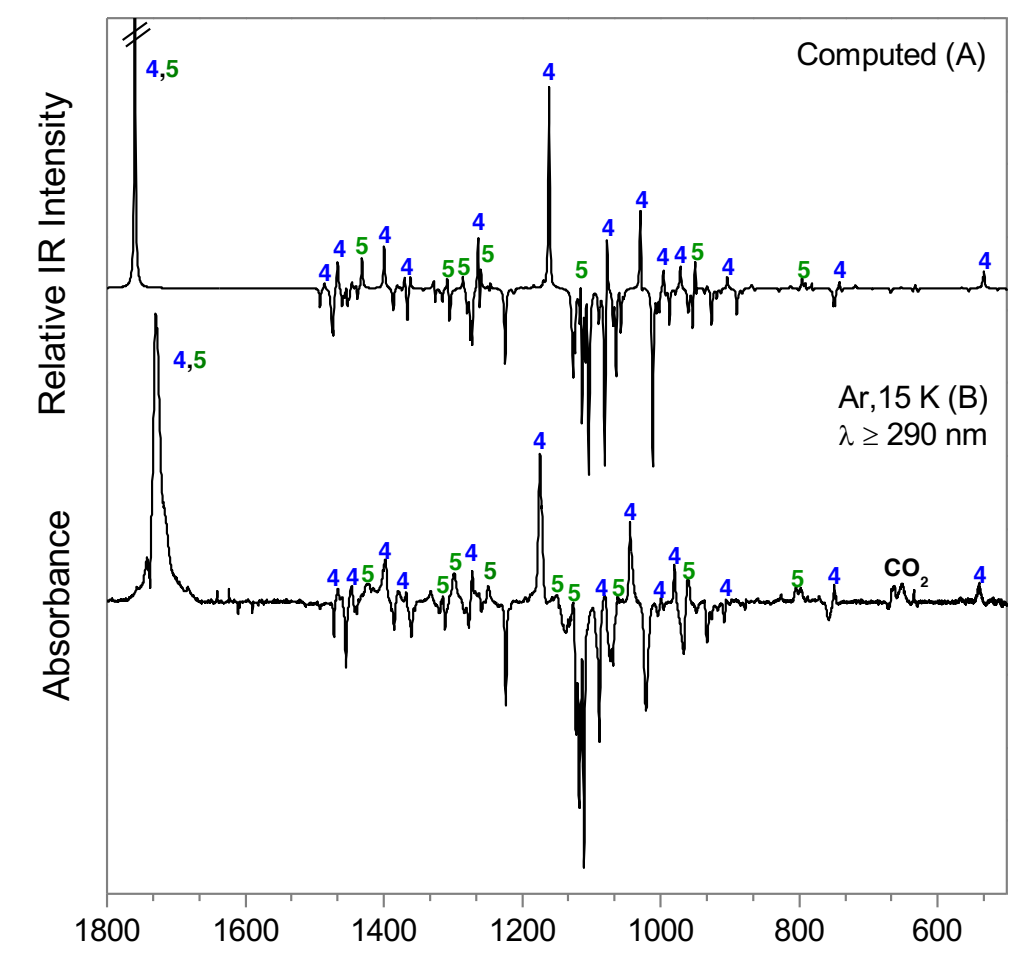

Wavenumber/ $\mathrm{cm}^{-1}$

316 Figure 3. (A) B3LYP/6-311++G(3df,3pd) calculated difference IR spectrum: spectra of 4-

317 oxahomoadamantan-5-one $\mathbf{4}$ and 1,4-cyclohexanedione $\mathbf{5}$ minus the spectrum of $\mathbf{1}$ [in a ratio 318 (0.5+0.5):1]. (B) Experimental difference IR spectrum: spectrum after irradiation at $\lambda \geq 290$ (30 min.; 319 Ar matrix at $15 \mathrm{~K}$ ) minus spectrum of the as-deposited matrix. The negative bands were assigned to $320 \mathbf{1}$; the positive ones were assigned to the new photogenerated species $\mathbf{4}$ and $\mathbf{5}$. 

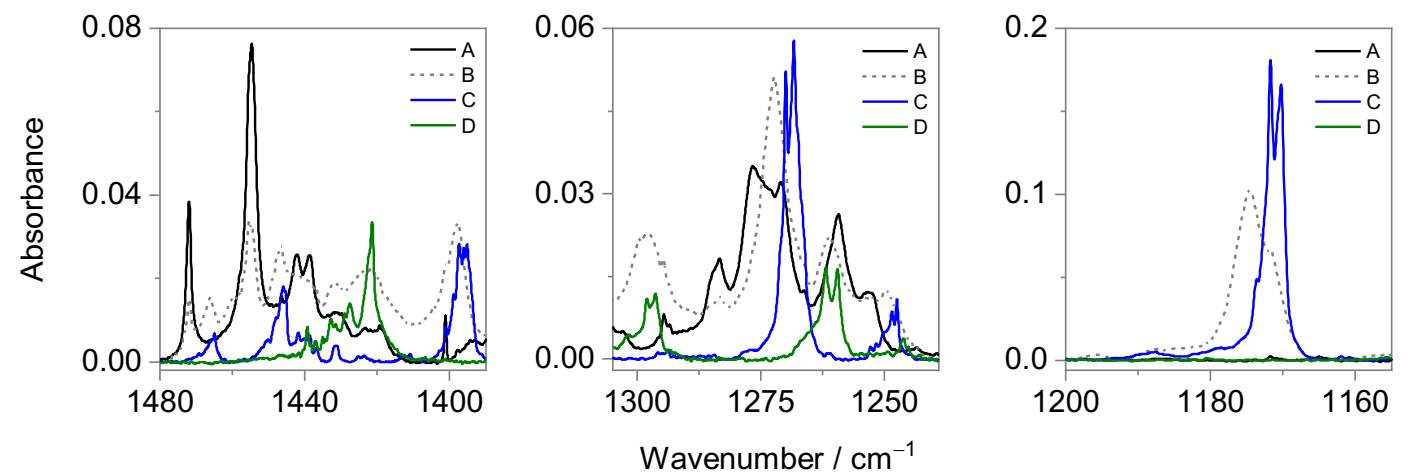

Figure 4. Comparison of selected regions of the experimental IR spectrum obtained before irradiation 324 of the matrix isolated $\mathbf{1}$ (Ar matrix at $15 \mathrm{~K}$ ) (solid black line; A) with the spectrum collected after 30 325 min of $\lambda \geq 290$ irradiation (dashed gray line; B) and with the spectra of authentic samples of 4326 oxahomoadamantan-5-one 4 (solid blue line; C) and 1,4-cyclohexanedione 5 (solid green line; D) 327 isolated in argon at $15 \mathrm{~K}$.

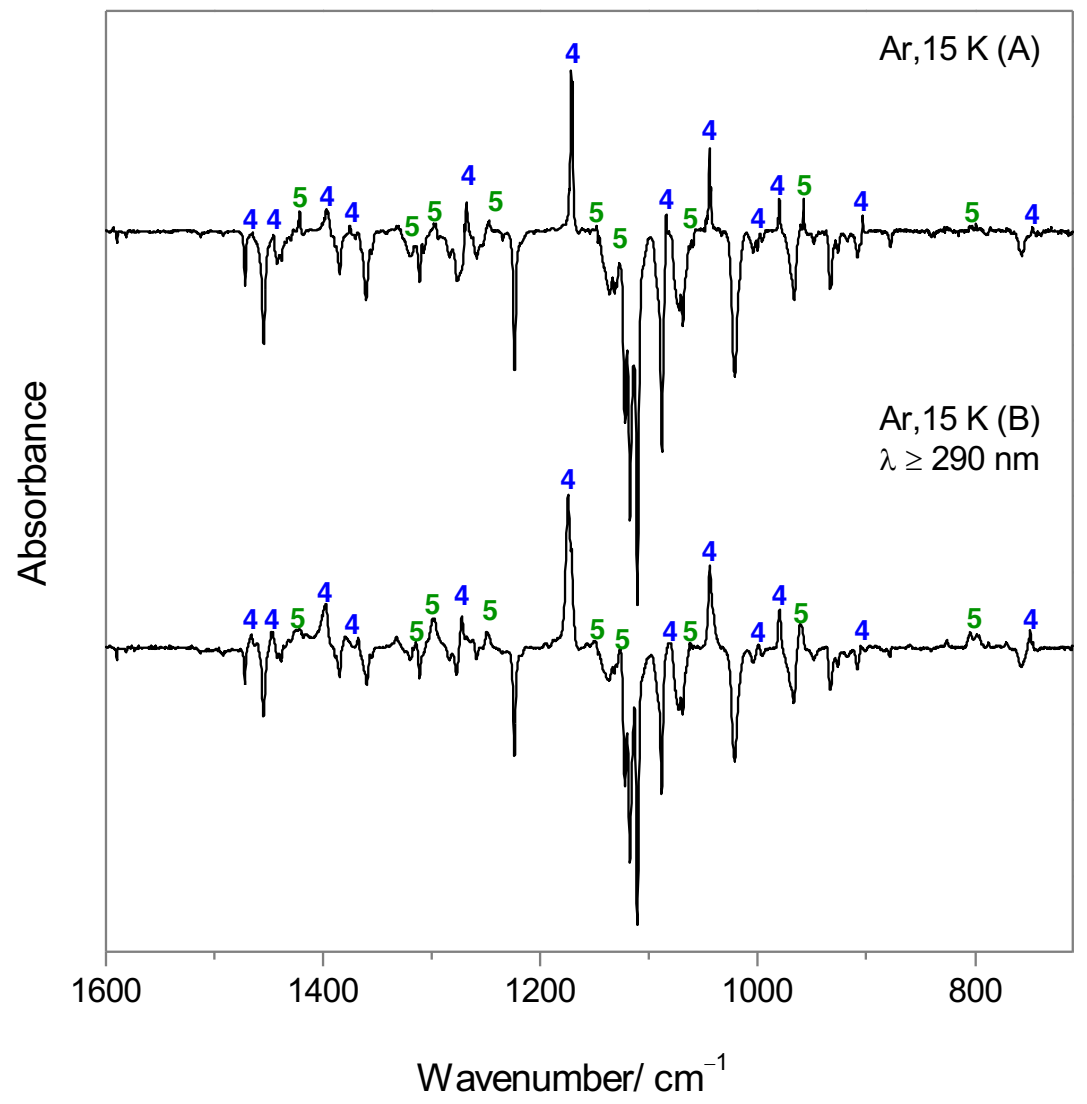

330 Figure 5. (A) Experimental difference IR spectrum: spectra of authentic samples of matrix isolated 331 4-oxohomoadamantan-5-one 4 and authentic 1,4-cyclohexanedione 5 minus spectrum of 1 Ar 
332 matrix at $15 \mathrm{~K}$ ). (B) Experimental difference IR spectrum: spectrum after irradiation of matrix 333 isolated $\mathbf{1}(\lambda \geq 290 ; 30 \mathrm{~min})$ minus spectrum of the as-deposited matrix (Ar matrix at $15 \mathrm{~K}$ ).

EPR detection of the dioxygen-centered 2 and carbon-centered/oxygen-centered 3 diradicals

337 The in situ EPR measurements in the photolysis of 1 in MeTHF matrix were conducted 338 using $266 \mathrm{~nm}$ laser light $(5 \mathrm{~mJ})$ at 10-50 K. The X-band EPR signals were measured at a 339 resonance frequency of $9.40 \mathrm{GHz}$ (Figure 6). During the photolysis at $10 \mathrm{~K}$ (Figure $6 \mathrm{Ib}$ ), a 340 half-field signal at $\sim 1550 \mathrm{G}$ (triplet species A) was observed after 120 seconds, which is a 341 typical triplet species of diradicals, and another half-field signal at $1670 \mathrm{G}$ (triplet species B) 342 was detected after 420 seconds, together with the $1550 \mathrm{G}$ signal (Figure $6 \mathrm{Ic}$ ). Both resonance 343 frequencies (1550 and $1670 \mathrm{GHz}$ ) are typical for triplet diradicals. Under dark conditions, 344 only the half-field signal at $1670 \mathrm{G}$ was detected (Figure 6 Id), indicating that the disappeared 345 triplet species A is short-lived, while the triplet species B is persistent, under the MeTHF 346 matrix conditions, at $10 \mathrm{~K}$. 


\section{I.}
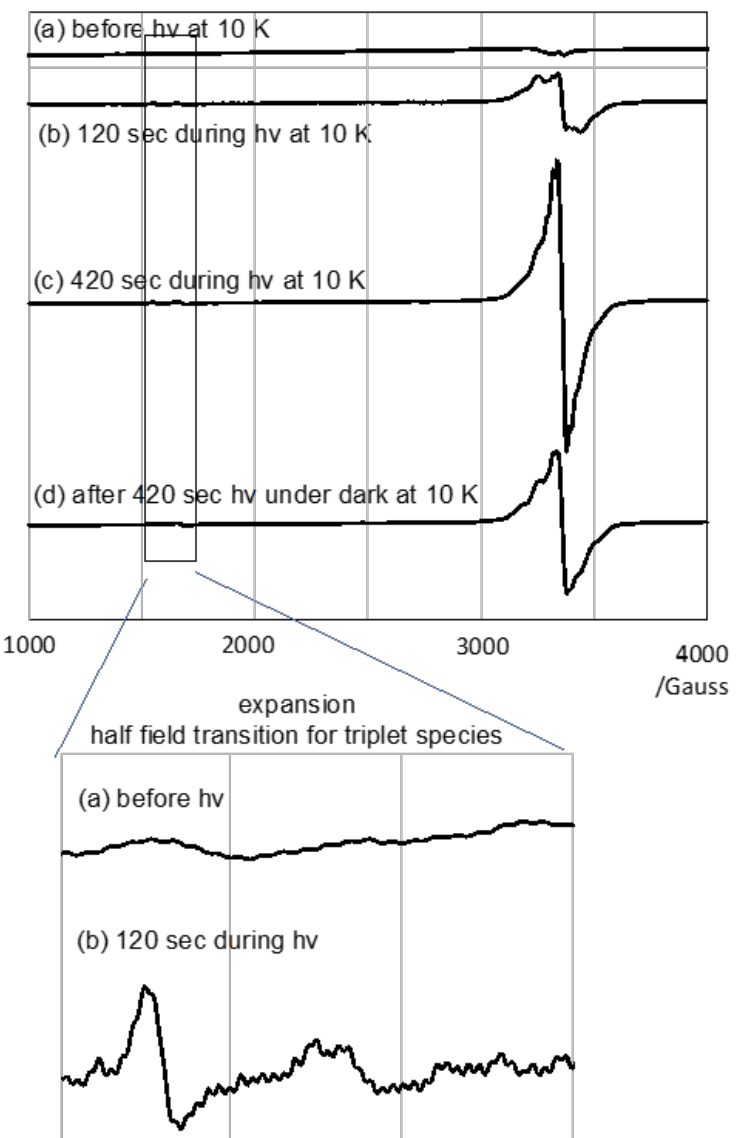

(c) 420 sec during hv

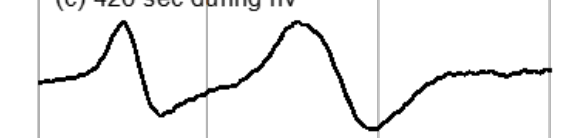

(d) after $420 \mathrm{sec}$ hv under dark

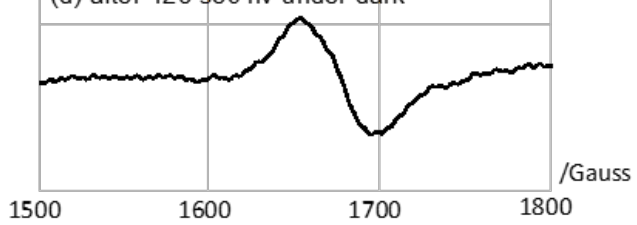

II.

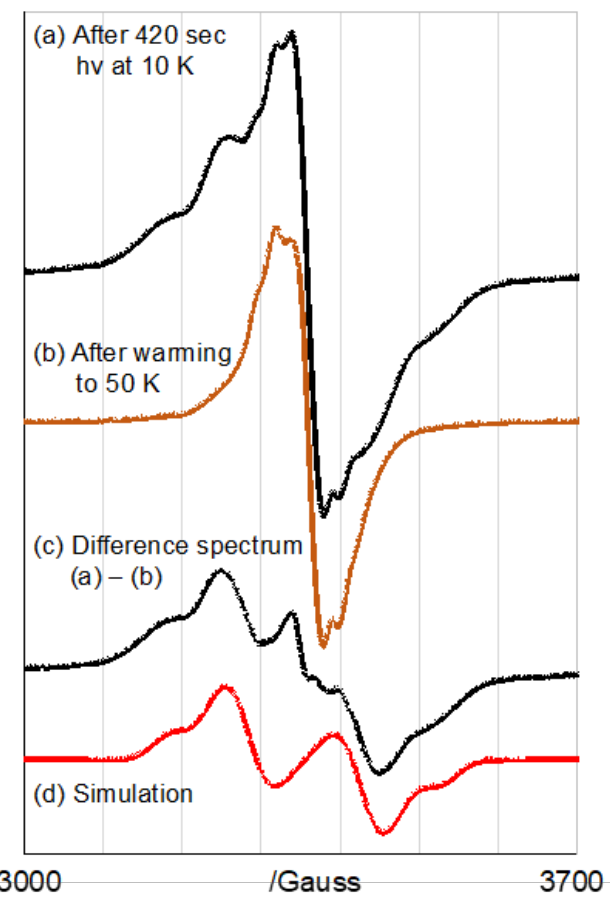

348 Figure 6. I: X-band EPR spectra $(9.40 \mathrm{GHz}, 1000-4000 \mathrm{G})$ obtained from photolysis studies of 349 compound $1(100 \mathrm{mM})$ in MeTHF-matrix, at $266 \mathrm{~nm}$; (a) before irradiation at $10 \mathrm{~K}$; (b) after 350 irradiation for 120 seconds at $10 \mathrm{~K}$; (c) after irradiation for 420 seconds, at $10 \mathrm{~K}$; (d) after irradiation 351 for 420 seconds, under dark, at $10 \mathrm{~K}$; II: X-band EPR signals (9.40 GHz, 3000-4000 G) from 352 photolysis of compound $1(100 \mathrm{mM})$ in MeTHF-matrix at $266 \mathrm{~nm}$; (a) after 420 seconds photolysis, 353 under dark, at $10 \mathrm{~K}$; (b) after warming the sample to $50 \mathrm{~K}$, and subsequent re-cooling to $10 \mathrm{~K}$; (c) 354 difference spectrum between (a)-(b); (d) simulated spectrum with $D / \mathrm{hc}=0.160 \mathrm{~cm}^{-1}$ and $E / \mathrm{hc}=0.001$ $355 \mathrm{~cm}^{-1}$ at $\mathrm{g}$ value of 2.003 . 
To gather further insight regarding the reactivity of the triplet species B, the photolystate at $10 \mathrm{~K}$ was warmed to $50 \mathrm{~K}$ under dark conditions (Figure $6 \mathrm{IIb}$ ). The typical triplet signal disappeared and did not recover to the original one in Figure 6 Ic after re-cooling the sample to $10 \mathrm{~K}$, indicating that the triplet species $\mathrm{B}$ was also thermally labile at $\sim 50 \mathrm{~K}$. The triplet signal of B was obtained by the difference spectrum of Figure 6 IIa-b (Figure 6 IIc). The zero-field splitting parameters $D /$ hc and $E /$ hc of the triplet species B were determined to be $0.160 \mathrm{~cm}^{-1}$ and $0.001 \mathrm{~cm}^{-1}$ after the simulation (Figure 6 IId). From the $D /$ hc value, the distance between two spins was estimated to be $\sim 5 \AA$.

The EPR results demonstrate that two triplet species (A and $\mathrm{B}$ ) are formed during the photolysis of the studied trioxolane 1, in agreement with the expectations. One of the triplet species (A) is thermally quite labile, while the other (B) is persistent at $10 \mathrm{~K}$. Considering 368 the results obtained in the photolysis of $\mathbf{1}$ in argon matrix, in particular the obtained final products, the theoretical data described in the previous sections, and the accumulated literature evidence, diradicals $\mathbf{2}$ and $\mathbf{3}$ shall be assigned to the intermediates $\mathrm{A}$ and $\mathrm{B}$, respectively, detected by EPR. This is the first experimental detection of these diradical

372 intermediates.

\section{CONCLUSIONS}

Adamantane-2-spiro-3'-8'-oxo-1',2',4'-trioxaspiro[4,5]decane 1, a reported potent antiparasitic dispiro-1,2,4-trioxolane, has been synthesized and studied from the view point of its molecular structure and monomeric photochemistry, using matrix isolation techniques coupled to FTIR and EPR spectroscopy. It is noteworthy that the ozonide 1 remained thermally stable upon sublimation required for matrix deposition. All experimental results 380 have been supported by quantum chemical calculations using two levels of theory [B3LYP/6$381311++\mathrm{G}(3 \mathrm{df}, 3 \mathrm{dp})$ and M06-2X/6-311++G(3df,3dp)], the comparison of the spectrum 382 obtained in the argon matrix with those theoretically predicted showing that the B3LYP 383 functional reproduces better the experimental data throughout the spectrum than the M06384 2X functional. Both methods predict two conformers for the dispiro-1,2,4-trioxolane 1 with 385 very close energies, differing in the orientation of the spiro-p-cyclohenanonyl moiety, which 386 in the most stable form is directed to the peroxide group, while in the less stable one is 387 oriented towards the ring ether fragment. In the experimental spectra, several bands were 
observed which permitted the assignment of the individual forms, thus confirming the presence of both conformers in the matrices.

The UV-induced reactivity of $\mathbf{1}$ was investigated in the two types of matrices $\left(\mathrm{Ar}, \mathrm{N}_{2}\right)$, using broadband $(\lambda \geq 290 \mathrm{~nm})$ and narrowband $(\lambda=290 \mathrm{~nm})$ irradiation, the results proving

392 qualitatively identical in both matrices and for the two followed excitation procedures.

393 Irradiation of matrix-isolated 1 at $\lambda \geq 290 \mathrm{~nm}$ resulted in photocleavage of the trioxolane 394 pharmacophore with formation of other chemical species, as evidenced by the observation of 395 new spectral features in the $v(\mathrm{C}=\mathrm{O})$, (between $\left.1720-1750 \mathrm{~cm}^{-1}\right)$ and $v(\mathrm{C}-\mathrm{O}),\left(1080-1120 \mathrm{~cm}^{-}\right.$ $396^{1}$ ) regions. Detailed analysis of the spectra of the photolyzed matrices allowed identification 397 of 4-oxahomoadamantan-5-one $\mathbf{4}$ and 1,4-cyclohexanedione 5 as the sole final products from 398 photolysis of the trioxolane 1. This identification was further confirmed through comparison 399 with the matrix spectra of the pure compounds. Such observation indicates that the dioxygen400 centered diradical 2 , formed upon homolytic cleavage of the labile peroxide bond, undergoes 401 a regioselective radical isomerization to form the more stable (secondary carbon402 centered)/oxygen-centered diradical 3. In fact, no evidence was observed for the formation 403 of 2-adamantanone and oxocane-2,7-dione, the products that would arise from the putative 404 alternative (primary carbon-centered)/oxygen-centered diradical. The observed 405 regioselectivity can be explained by the expected greater stability of the (secondary carboncentered)/oxygen-centered diradical species $\mathbf{3}$ in comparison to its primary counterpart. Spin 407 density calculations (performed at the B3LYP/6-311++G(3df,3dp) level) on the triplet state 408 dioxygen-centered diradical result in a considerably larger electron spin density in the tertiary $409 \beta$-carbon of the spiroadamantyl substituent as compared to that of the secondary $\beta$-carbon of 410 the spiro- $p$-cyclohexanonyl substituent, which can also be taken as an indication of a most 411 favorable rearrangement of the initially generated dioxygen-centered diradical into the 412 (secondary carbon-centered)/oxygen-centered diradical species $\mathbf{3}$, leading to the final 413 products identified. These results are in keeping with information gathered from studies on 414 the mechanisms of bioactivation and action of trioxolane based antiparasitic candidates, 415 where heme-adducts of the secondary carbon-centered)/oxygen-centered diradical species 416 analogues of $\mathbf{3}$ were detected ${ }^{47}$.

417 In situ EPR measurements during photolysis of compound 1 deposited in a MeTHF418 matrix led to the detection of signals at1550 and $1670 \mathrm{GHz}$, corresponding to resonances of 
419 two triplet diradicals, the one at $1550 \mathrm{GHz}$ appearing thermally labile while the other (1670

$420 \mathrm{GHz}$ ) proved persistent at $10 \mathrm{~K}$. The observation of signals with resonance frequencies that

421 are typical for triplet diradicals, combined with the product characterizations achieved

422 through the matrix isolation FTIR studies, indicate that the postulated diradicals $\mathbf{2}$ and $\mathbf{3}$ shall

423 correspond to the intermediates detected by the experiment.

424 In conclusion, our results support the proposal of a regioselective radical isomerization 425 of dioxygen-centered diradical 2 to afford the (secondary carbon-centered)/oxygen-centered 426 diradical $\mathbf{3}$, also providing evidence for the presence of both intermediate diradicals on a 427 photolyzed MeTHF-matrix containing compound 1. This work deepens the knowledge 428 concerning the photoreactivity/photostability of antiparasitic endoperoxides based on the 429 trioxolane pharmacophore.

\section{ASSOCIATED CONTENT}

432 Supporting Information. Supplemental figures and calculated data for adamantane-2-spiro433 3'-8'-oxo-1',2',4'-trioxaspiro[4,5]decane. This material is available free of charge via the 434 Internet at http://pubs.acs.org

436 AUTHOR INFORMATION

437 Corresponding Author

$438 *$ E-mail: rfausto@ci.uc.pt

439 *E-mail: mcristi@ualg.pt

$440 \quad$ Notes

441 The authors declare no competing financial interest.

442 Author Contributions

443 The authors contributed equally.

\section{ACKNOWLEDGEMENTS}

448 The Coimbra Chemistry Centre (CQC) and the Center of Marine Sciences (CCMar) are

449 supported by the Portuguese Science Foundation (FCT; Projects UID/QUI/0313/2019 and 
UID/MULTI/04326/2019) and COMPETE-EU. E.M.B. thanks FCT for both the Research

451 Grants CCMAR/BI/0013/2017, awarded within the Project PTDC/MAR-BIO/4132/2014, and SFRH/BD/136246/2018. L.I.L.C. thanks FCT and CCMar for the Research Grant CCMAR/BI/0017/2016 awarded within the Project PTDC/MAR-BIO/4132/2014. P.S.M.A. 454 thanks FCT for the Research Grant SFRH/BD/130407/2017.

\section{REFERENCES}

1. Tu, Y. Y. The discovery of artemisinin (qinghaosu) and gifts from Chinese medicine, Nature Med., 2011, 17, 1217-1220.

2. Tu, Y. Y. Artemisinin - A Gift from Traditional Chinese Medicine to the World (Nobel Lecture). Angew. Chem. Int. Ed., 2016, 55, 10210-10226.

3. Liu, C. X. Discovery and Development of Artemisinin and Related Compounds. Chin. Herb. Med., 2017, 9, 101-114.

4. Jefford C. W. Why Artemisinin and Certain Synthetic Peroxides are Potent Antimalarials. Implications for the Mode of Action. Curr. Med. Chem., 2011, 8, 1803-1826.

5. Cumming, J. N.; Polypradith, P.; Posner, G. H. Antimalarial Activity of Artemisinin (Qinghaosu) and Related Trioxanes: Mechanism(s) of Action. Adv. Pharmacol., 1996, 37, 253-297.

6. Wu, Y. How Might Qinghaosu (Artemisinin) and Related Compounds Kill the Intraerythrocytic Malaria Parasite? A Chemist's View. Acc. Chem. Res., 2002, 35, 255-259.

7. Robert, A.; Coppel, Y.; Meunier, B. Alkylation of heme by the antimalarial drug artemisinin. Chem. Commun., 2002, 414-415.

8. Meshnick, S. R.; Artemisinin: mechanisms of action, resistance and toxicity. Int. J. Parasitol., 2002, 32, 1655-1660.

9. Eckstein-Ludwig, U.; Krieger, J.; Smeilus, T.; Kaiser, M.; Seo, E. J.; Efferth, T.; Ginannis, A. Total Synthesis and Biological Investigation of (-)-Artemisinin: The Antimalarial Activity of Artemisinin Is not Stereospecific. Angew. Chem. Int. Ed., 2018, 57, 8293-8296. 
10. Tilley, L.; Straimer, J.; Gnädig, N. F.; Ralph, S. A.; Fidock, D. A. Artemisinin Action and Resistance in Plasmodium falciparum. Trends Parasit., 2016, 32, 682-696.

11. Winzeler, E. A.; Manary, M. J. Drug resistance genomics of the antimalarial drug artemisinin. Gen. Biol., 2014, 15, 544.

12. Cravo, P.; Napolitano, H.; Culleton, R. How genomics is contributing to the fight against artemisinin-resistant malaria parasites. Acta Tropica, 2015, 148, 1-7.

13. Wang, J.; Zhang, C.; Chia, W.; Loh, C.; Li, Z.; Lee, Y.; He, Y.; Yuan, L.; Lim, T.; Liu, M. et al. Haem-activated promiscuous targeting of artemisinin in Plasmodium falciparum. Nature Comm., 2015, 6, Art. № 10111 (1-11).

14. Aweeka, F. T.; German, P. I. Clinical Pharmacology of Artemisinin-Based Combination Therapies. Clin. Pharmacokin., 2008, 47, 91-102.

15. Sugimone, H. H. In Handbook of Organic Photochemistry and Photobiology. Horspool, W. M.; Song, P. S. Eds.; CRC Press, London, 1994, pp 1229-1253 references therein.

16. Jefford, C. W.; Rossier, J. C.; Boukouvalas, J. Eliminative ring fission of 1,2,4trioxan-5-ones. A new approach to $\alpha$-keto acids. J. Chem. Soc. Chem. Commun., 1986, 1701-1702.

17. Jefford, C. W.; Rossier, J. C.; Boukouvalas, J. A Mild and Efficient Preparation of cis-1,2-Diols from 1,2,4-Trioxanes. J. Chem. Soc. Chem. Commun., 1987, 15931594.

18. Jefford, C. W.; Kohmoto, S.; Rossier, J. C.; Boukouvalas, J. Chemistry of 1,2,4Trioxanes. Formation of 1,2-Diol Monoesters. J. Chem. Soc. Chem. Commun., 1985, 1783-1784.

19. Jefford, C. W.; Rossier, J. C.; Boukouvalas, J. 1,2,4-Trioxanes as masked, dual purpose, functional groups. Heterocycles, 1989, 28, 673-676.

20. Jefford, C. W.; Rossier, J. C.; Boukouvalas, J. Electrophile-induced rearrangement of 1,2,4-trioxanes. Formation of 1-benzofuran and 2H-1-benzopyrans. J. Chem. Soc. Chem. Commun., 1987, 713-714.

21. Erhardt, S.; Macgregor, S. A.; McCullough, K. J.; Savill, K.; Taylor, B. J. Model Studies of $\beta$-Scission Ring-Opening Reactions of Cyclohexyloxy Radicals: 
Application to Thermal Rearrangements of Dispiro-1,2,4-trioxanes. Org. Lett., 2007, 9, 5569-5572.

22. Tang, Y.; Dong, Y.; Wang, X.; Sriraghavan, K.; Wood, J. K.; Vennerstrom J. L. Dispiro-1,2,4-trioxane Analogues of a Prototype Dispiro-1,2,4-trioxolane: Mechanistic Comparators for Artemisinin in the Context of Reaction Pathways with

23. Haq, A.; Kerr, B.; McCullough K. J. A Rapid Route to Medium to Large Ring Lactones via the Thermolysis of Dispiro-1,2,4-trioxane Derivatives. J. Chem. Soc. Chem. Commun., 1993, 1076-1078.

24. Abe, M.; Adam, W.; Heidenfelder, T.; Nau, W. M.; Zhang, X.; Intramolecular and Intermolecular Reactivity of Localized Singlet Diradicals: The Exceedingly LongLived 2,2-Diethoxy-1,3-diphenylcyclopentane-1,3-diyl. J. Am. Chem. Soc., 2000, $122,2019-2026$.

25. Abe, M.; Inakazu, T.; Munakata, J.; Nojima, M. ${ }^{18} \mathrm{O}-$ Tracer Studies of Fe(II)-Induced Decomposition of 1,2,4-Trioxolanes (Ozonides) Derived from Cyclopentenes and Indenes. Inner-Sphere Electron Transfer Reduction of the Peroxide Linkage. J. Am. Chem. Soc., 1999, 121, 6556-6562.

26. Meyer, B. Low Temperature Spectroscopy, American Elsevier Publishers Company; New York (USA), 1971.

27. Andrews L.; Moskovits, M. Chemistry and Physics of Matrix Isolated Species. Eds., Elsevier, Amsterdam (Holland), 1989.

28. Barnes, A.; Orville-Thomas, W. J.; Gaufrhs, R.; Muller, A. Matrix Isolation Spectroscopy. Eds.; Springer; Dordrecht (Holland), 1981.

29. Dunkin, I. R. Matrix Isolation Techniques: A Practical Approach. Oxford University Press; Oxford (UK), 1998.

30. Fausto, R. Low Temperature Molecular Spectroscopy. Ed., NATO-ASI Series C483; Kluwer, Amsterdam (Holland), 1996.

31. Vennerstrom, J. L.; Dong, Y.; Chollet, J.; Matile, H. Spiro and dispiro 1,2,4trioxolane antimalarials. U.S. patent. 6,486,199B1, 2002.

32. Lobo, L.; Cabral, L. I. L.; Sena, M. I.; Guerreiro, B. Rodrigues, A. S .; Andrade-Neto, V.F.; Cristiano, M. L. S.; Nogueira, F. New endoperoxides highly active in vivo and 
in vitro against artemisinin-resistant Plasmodium falciparum. Malar. J., 2018, 17, 111.

33. Cortes, S.; Albuquerque, A.; Cabral, L. I. L.; Lopes, L.; Campino, L.; Cristiano, M. L. S. In vitro susceptibility of Leishmania infantum to Artemisinin derivatives and selected trioxolanes. Antimicrob. Agents Chemother. 2015, 59, 5032-5035.

34. Araujo, N.C.P.; Afonso, R.; Beringela, A.; Cancela, M.L.; Cristiano, M.L.S.; Leite, R.B. Peroxides with antiplasmodial activity inhibit proliferation of Perkinsus olseni, the causative agent of Perkinsosis in bivalves. Parasitol. Int., 2013, 62, 575-582.

35. Renoud-Grappin, M.; Vanucci, C.; Lhommet, G. Diastereoselective synthesis of a limonoid model related to the insect antifeedant genudin. J. Org. Chem., 1994, 59, 3902-3905.

36. Raghavachari, K.; Binkley, J. S.; Seeger, R.; Pople, J. A. Selfconsistent molecular orbital methods. XX. A basis set for correlated wave functions. J. Chem. Phys., 1980, $72,650-654$.

37. Frisch, M. J.; Pople, J. A.; Binkley, J. S. Selfconsistent molecular orbital methods 25. Supplementary functions for Gaussian basis sets, J. Chem. Phys., 1984, 80, 3265-69.

38. Becke, A. D. Density-functional thermochemistry. III. The role of exact exchange. $J$. Chem. Phys., 1993, 98, 5648-5652.

39. Lee, C.; Yang, W.; Parr, R. G. Development of the Colic-Salvetti correlation-energy formula into a functional of the electron density. Phys. Rev. B, 1988, 37, 785-789.

40. Vosko, S. H.; Wilk, L.; Nusair, M. Accurate spin-dependent electron liquid correlation energies for local spin density calculations: a critical analysis. Can. J. Phys., 1980, 58, 1200-1211.

41. Stephens, P. J.; Devlin, F. J.; Chabalowski, C. F.; Frisch, M. J. F. Ab Initio Calculation of Vibrational Absorption and Circular Dichroism Spectra Using Density Functional Force Fields. J. Phys. Chem., 1994, 98, 11623-11627.

42. Zhao, Y.; Truhlar, D. G. The M06 suite of density functionals for main group thermochemistry, thermochemical kinetics, noncovalent interactions, excited states, and transition elements: two new functionals and systematic testing of four M06-class functionals and 12 other functionals. Theor. Chem. Acc., 2008, 120, 215-241. 
43. Zhao, Y.; Truhlar, D. G. Density Functionals with Broad Applicability in Chemistry. Acc. Chem. Res., 2008, 41, 157-167.

44. Frisch, M. J.; Trucks, G. W.; Schlegel, H. B.; Scuseria, G. E.; Robb, M. A.; Cheeseman, J. R.; Scalmani, G.; Barone, V.; Mennucci, B.; Petersson, G. A. et al. Gaussian 09, Revision D.01; Gaussian, Inc.; Wallingford, CT, 2004.

45. Lopes, S.; Domanskaya, A. V.; Fausto, R.; Räsänen, M.; Khriachtchev, L. Formic and acetic acids in a nitrogen matrix: Enhanced stability of the higher-energy conformer. J. Chem. Phys., 2010, 133, 144507.

46. Kus, N.; Fausto R. Effects of the matrix and intramolecular interactions on the stability of the higherenergy conformers of 2-fluorobenzoic acid. J. Chem. Phys., 2017, 146, 124305 .

586

587 